\title{
El desarrollo de capacidades de innovación. Un estudio del cluster de transporte en Aguascalientes, México ${ }^{\dagger}$
} The development of innovation capabilities. A study of the transport cluster in Aguascalientes, México

\section{O desenvolvimento de capacidades de inovaçáo. Um estudo do cluster de transporte em Aguascalientes, México}

\author{
Dra. Maribel Feria Cruz*
}

\section{RESUMEN}

La importancia de este trabajo radica en identificar la formación y consolidación de la capacidad innovativa de las pymes del cluster del transporte en contextos territoriales, caracterizando su nivel de desarrollo. El propósito del estudio es mostrar evidencia de la conformación del conglomerado mediante la forma en que las empresas operan, vinculan y construyen su capacidad innovativa en torno a los procesos de innovación. Los resultados arrojan problemas para generar procesos de desarrollo local virtuosos. Se recomienda la articulación de una oferta integral de servicios y estímulos de apoyo a la innovación en cada sector.

Palabras clave: cluster, capacidad de innovación, competitividad y relaciones de cooperación y colaboración.

\section{ABSTRACT}

The importance of this work is to identify the formation and consolidation of the innovative capability of SMEs cluster of transport in territorial contexts, characterizing their level of development. The purpose of the study is to show evidence of the formation of the conglomerate by way businesses operate, link and build their innovative capacity around innovation processes. The results show trouble generating virtuous local development processes. The articulation of a comprehensive range of services and incentives to support innovation in each sector is recommended.

Keywords: cluster, innovation capability, competitiveness and relations of cooperation and collaboration.

\section{RESUMO}

A importância deste trabalho radica na identificação da formaçáo e consolidação da capacidade de inovaçáo das pymes do cluster do transporte em contextos territoriais, caracterizando seu nível

† El presente artículo es resultado de una actualización de la investigación científica y tecnológica titulada "El cluster del transporte. Un estudio de caso dentro del Sistema Regional de Innovación”. Clave: AGS-MCA-2008-204. Por parte del Tecnológico Nacional de México.

* Mexicana, Dra. en Ciencias Sociales, adscrita a la Maestría en Ciencias en Administración y Licenciatura en Administración, Instituto Tecnológico de Aguascalientes, Aguascalientes, México. Correspondencia con la autora: fericruz@outlook.com. 
El desarrollo de capacidades de innovación. Un estudio del cluster de transporte en Aguascalientes, México / M. Feria

de desenvolvimento. O objetivo do estudo é mostrar evidência da conformação do conglomerado mediante a forma com que as empresas operam, vinculam e constroem sua capacidade de inovação em torno aos processos de inovaçáo. Os resultados mostram problemas para gerar processos de desenvolvimento local virtuosos. Recomenda-se a articulação de uma oferta integral de serviços e incentivos para apoiar a inovação em cada setor.

Palavras-chave: cluster, capacidade de inovação, competitividade e as relaçóes de cooperação e colaboração.

\section{Introducción}

El desarrollo económico en el mundo responde a cambios en las estructuras sociales y específicamente a los movimientos del mercado, las nuevas formas en que se conforman las estrategias productivas son el elemento clave para responder a las necesidades de los consumidores, es por estas circunstancias que surgen los complejos productivos llamados clusters. Estos conglomerados industriales pueden ser la respuesta a la búsqueda de mayores niveles de innovación y competitividad de las empresas a través de un proceso de integración y asociatividad entre cada uno de los elementos que conforman la agrupación. En este escenario marcadamente global, la capacidad endógena tecnoproductiva de los agentes y el grado de desarrollo del territorio en el que actúan se han convertido en elementos clave para la construcción de ventajas competitivas (Boscherini y Poma, 2000), y donde la capacidad de innovación cobra vital importancia.

\section{Referente Teórico: capacidades de innovación como fuente de competitividad}

El nuevo esquema competitivo torna importante la manera como las empresas responden ante esta situación y cómo idean, planifican y realizan desarrollos y mejoras de productos y procesos, que los llevan a cambios organizativos dirigidos a nuevas formas de vinculación en el mercado. Es decir, los agentes del sistema tratan de aumentar su capacidad innovativa, entendida como la potencialidad de los agentes para transformar conocimientos genéricos en específicos a partir de su acervo de competencias, lo que involucra aprendizajes formales e informales, codificados y tácitos (Ernest y Lundvall, 1997; Boscherini y Yoguel, 1996).

Las capacidades de innovación son fuente de competitividad de las empresas (Leonard, 1995; Teece et al., 1997 y Dosi et al., 2000). Leonard (1995) introduce el término Core Capabilities definiéndolo como el sistema de actividades, sistemas físicos, habilidades y conocimiento, así como los sistemas gerenciales, de educación y recompensa, incluyendo 
los valores que crean una ventaja especial para una institución. Teece et al. (1997) empleó el término "Dynamic Capabilities", para referirse a la habilidad de la firma para integrar, construir y configurar competencias tanto internas como externas y poder enfrentar de una manera rápida y eficiente al medio ambiente. Asimismo, Dosi, Nelson y Winter (2000) definen a las Organizational Capabilities como el know how que capacita a las organizaciones para desempeñar ciertas actividades como la creación de un producto tangible o la provisión de un servicio y el desarrollo de nuevos productos y servicios. Estas capacidades dependen de los niveles de habilidad y de comunicación efectiva, tanto fuera como dentro de la empresa. Para estos autores, las capacidades de innovación se encuentran embebidas dentro de las habilidades de los actores; las mismas sirven para emplear de manera eficiente tanto sus competencias internas (aprendizaje tecnológico y modos de construcción del conocimiento) como sus competencias externas, las cuales van dirigidas (implícita pero no necesariamente) al uso del conocimiento externo a través del establecimiento de redes de relaciones sociales que la empresa establece con otras instituciones: proveedores, usuarios, universidades, centros de investigación, etc., no perdiendo de vista su identidad cultural (sus elementos sociales y culturales).

En este sentido, las capacidades de innovación pueden proveer ventajas competitivas si están basadas sobre una sucesión de rutinas, habilidades que son difíciles de imitar o transferir, y sólo puede entenderse en su conjunto lo que adquiere sentido contextualmente. Es decir, para comprenderla se necesita conocer todo el contexto donde actúan o se construyen, por eso se habla de un entorno específico y sistémico.

La innovación es un elemento fundamental en el desarrollo de cualquier actividad humana, innovar no es simplemente cambiar, implica evolucionar y mejorar, obtener mejores resultados a partir de modificaciones conscientes; en términos de desarrollo industrial, la innovación puede ser definida como la fuerza impulsora que propicia mayores niveles de eficiencia y competitividad empresarial. La innovación tiene que ver con la generación y gestión del conocimiento, y consecuentemente con el proceso de aprendizaje. Las capacidades de innovación son construidas a lo largo del tiempo y no son fáciles de codificar, por lo que su análisis exige un patrimonio de competencias (su reconstrucción a través del tiempo).

\section{La formación de clusters como estrategia competitiva}

Los cambios que se han producido en las últimas décadas han provocado importantes transformaciones en el escenario mundial, lo cual ocasiona un aumento de la presión competitiva y de las incertidumbres estratégicas que enfrentan los agentes. Los cambios en los paradigmas tecnoorganizativos, intensivos en información y conocimiento han acelerado la globalización de los mercados y sobre todo la competitividad (Feria, 2005). 
El desarrollo de capacidades de innovación. Un estudio del cluster de transporte en Aguascalientes, México / M. Feria

La consecuencia que provoca este conjunto de cambios conduce también a que la concepción anterior de la competitividad entre en crisis, considerada como un fenómeno exclusivamente de naturaleza macroeconómica determinada por ventajas comparativas estáticas o influida por la dotación factorial. Las políticas industriales del Gobierno del estado de Aguascalientes han estado enfocadas al desarrollo de la competitividad de la planta productiva estatal y la formación de clusters ha sido una de las estrategias aplicadas para dicho fin.

Los clusters se definen como "una aglomeración territorial de industrias estrechamente relacionadas entre sí, y en su mayoría nacen debido a una coincidencia histórica” (Meyer-Stamer y Harmes-Liedtke, 2005:1).

A nivel internacional el desarrollo de clusters ha significado un incremento en los niveles de innovación y competitividad, por ello es importante realizar un diagnóstico de la situación, de la manera en que establecen sus relaciones las diversas empresas que conforman el cluster del transporte en Aguascalientes, mediante la construcción de su capacidad innovativa desde la perspectiva de su integración en redes, y en este caso, la manera en que las empresas del cluster contribuyen a construir esa capacidad innovativa en el estado.

Así, el presente estudio se ubica en el estado de Aguascalientes; el objetivo del trabajo fue tratar de identificar la manera en que establecen sus relaciones las diversas empresas que conforman el cluster del transporte de Aguascalientes, desde su perspectiva de su integración en redes y el nivel de desarrollo del mismo en cuanto a su forma de operar y vincularse, de manera que sus esfuerzos se dirijan hacia la construcción de la capacidad innovativa de este conglomerado, a fin de caracterizarlo, detectar su nivel de desarrollo y apuntar algunas causas de sus problemas, sus debilidades y fortalezas. La pregunta básica que subyace bajo estos grandes lineamientos es: ¿cuál es la relación que existe entre las empresas que integran el cluster del transporte en Aguascalientes y su nivel de desarrollo, en cuanto a la forma de operar y a la manera como se vinculan para que generen la construcción de capacidades de innovación necesarias para su desempeño innovativo y por ende sean más competitivas?

La hipótesis inicial parte de que existe una relación significativa entre las empresas que integran el cluster del transporte en Aguascalientes y su nivel de desarrollo en cuanto a su manera de operar y vincularse, pero que esta relación presenta un bajo nivel de integración y por ende una baja capacidad innovativa, lo que está relacionado con bajos niveles de desarrollo de sus entrelazamientos en red, debido al comportamiento desigual de los actores respecto a varios factores como son: el conocimiento y aprovechamiento del sistema de incentivos e instrumentos de apoyo; la capacidad de interacción de todos los actores para producir, adaptar, gestionar, intercambiar y difundir el conocimiento, 
así como para desarrollar las capacidades tecnológicas necesarias a la creación de un entorno favorable.

El presente trabajo expone, pues, los resultados y evidencias que apoyan la hipótesis planteada, a efectos de lo cual se estructura de la siguiente manera: el apartado 1 presenta de manera condensada el estado del conocimiento en este campo de estudios; el apartado 2 describe los procedimientos metodológicos seguidos para someter a prueba las hipótesis; el apartado 3 presenta el análisis de los datos de campo y discute los resultados obtenidos; el último de ellos, el 4, menciona las conclusiones que se desprenden de la problemática abordada.

\section{Panorama general de los clusters}

La estrategia empresarial conocida como cluster ha sido utilizada exitosamente en diversas regiones del mundo y bajo condiciones socio-geográficas y económicas muy diferentes: Alemania, Italia, Francia y España, en Europa, y en América, Estados Unidos es el ejemplo más claro de éxito empresarial a través de aglomeraciones industriales. En Europa, la región de Emilia-Romagna (Italia) "ocupa el undécimo lugar entre las regiones de la Unión Europea en cuanto a ingresos per cápita; es una de las más altas, y la tasa de desempleo es una de las más bajas. Baden-Wuerttemberg (Alemania), produce el 17\% de las exportaciones alemanas (Mercedes Benz y Porsche). Cataluńa, es el centro industrial de España, genera el 20\% del PIB español y representa el 27\% del total del rendimiento industrial de este país. Silicon Valley en California, USA, tiene 6000 empresas en las que trabajan un millón de personas, la mayoría en el desarrollo de microelectrónica y computadoras" (Herrera, 2006).

La idea básica de especialización industrial determinada por áreas geográficas fue senáada en el campo de la teoría económica por Adam Smith y ampliada por Marshall (1920), en sus estudios sobre distritos industriales en Inglaterra y posteriormente desarrollada por Porter (1991). Las empresas localizadas en clusters geográficos o distritos industriales se benefician competitivamente a través del acceso a habilidades e inputs especializados y de procesos de retroalimentación del conocimiento entre las mismas. Existen diferentes definiciones de clusters, dependiendo del enfoque o disciplina que estudie, el concepto tendrá acepciones diferentes; para efectos del siguiente trabajo se considerará la teoría económica para definir dichos agrupamientos industriales. Los clusters se definen como "una aglomeración en un territorio específico donde las industrias se relacionan estrechamente entre sí, y en su mayoría nacen debido a un conjunto de situaciones de carácter histórico social" (Meyer-Stamer y HarmesLiedtke, 2005:8). 
El desarrollo de capacidades de innovación. Un estudio del cluster de transporte en Aguascalientes, México / M. Feria

También puede entenderse por cluster "una concentración geográfica y/o sectorial de empresas en las mismas actividades o en actividades estrechamente relacionadas, con importantes y acumulativas economías externas, de aglomeración y especialización de productores, proveedores y mano de obra especializada, de servicios anexos específicos al sector, con la posibilidad de acción conjunta en búsqueda de eficiencia colectiva” (Ramos, 1998:4).

Los clusters son una nueva forma de organización de la cadena de valor que se encuentra situada entre la mano del mercado, por un lado, y jerarquías organizacionales o integración vertical, por el otro. La proximidad local de companías e instituciones, y el establecimiento de relaciones entre ellas, procura una mayor coordinación y confianza que la simple interacción de mercado entre actores dispersos geográficamente. La coordinación y confianza entre organizaciones son mucho más flexibles que las que proveen las integraciones verticales o las relaciones formales entre empresas como redes, alianzas o colaboraciones (Porter, 1998).

Existen características específicas para identificar un cluster o complejo productivo: se puede decir que es un "agrupamiento de empresas, principalmente de pequeña y mediana dimensión, concentradas geográficamente y con una especialización sectorial, vinculadas hacia delante y hacia atrás, basadas en relaciones de mercado y extra-mercado para el intercambio de bienes, información y recursos humanos, también es necesario un entorno cultural y social común que vincule de manera efectiva a los agentes económicos y permita la creación de códigos de comportamiento comunes implícitos y explícitos; además, una red de instituciones públicas y privadas locales de apoyo vinculadas a los agentes económicos" (Rabellotti,1998:14).

El grupo de la OCDE (1992 y 1999) centrado en el análisis de clusters lo define como: "la red de producción de empresas fuertemente interdependientes (que incluye proveedores especializados) vinculadas entre sí en una cadena de producción que añade valor. Los clusters también comprenden alianzas estratégicas con universidades, institutos de investigación, servicios empresariales intensivos en conocimiento, instituciones puentes (comisionistas, consultores) y clientes" (OECD, 1999:85).

De la misma forma en la que existen diferentes definiciones de clusters, así también se tienen diversos enfoques o tesis que explican el porqué de la formación de dichas aglomeraciones industriales. Entre las principales características sobre las que se basan estas teorías se pueden señalar: la ubicación o situación geográfica, la forma de integración interempresarial, la competitividad, y la dotación de factores naturales (Perego, 2003).

Asimismo, Osorio (2006:37) señala que entre los factores fundamentales en la formación de clusters se encuentra la generación de redes sociales, ya que a partir de ellas se va 
conformando el sistema de competitividad cuya importancia radica en que se reducen los costos de transacción, por la vía de la internalización de las transacciones al interior de las redes, mediante el principio de la cercanía.

\section{Dimensión territorial y carácter local de la difusión de conocimiento}

El contexto territorial tiene una fuerte influencia sobre la evolución y difusión del conocimiento y las innovaciones. El carácter tácito de la difusión de conocimiento, es el conjunto de elementos difíciles de codificar y, por lo mismo, de difundir formalmente. Cuando estos elementos tácitos se incrementan dentro de la base de conocimiento, la acumulación tecnológica se empieza a basar más en la experiencia y en los contactos interpersonales. Además de las diferentes interrelaciones que se crean entre todos los integrantes del cluster y de la influencia del factor territorial, es en la difusión de conocimientos que existen alicientes que fomentan las integraciones industriales, los principales incentivos para la formación de clusters y alianzas han sido la reducción de los costes de transacción, el desarrollo de nuevas habilidades, la superación (o creación) de barreras de entrada en los mercados y la aceleración del proceso de aprendizaje (Scott y Storper, 2003).

La teoría de los encadenamientos hacia atrás y hacia adelante de Hirschman (1958), procura demostrar cómo y cuándo la producción de un sector es suficiente para satisfacer el umbral mínimo o escala mínima necesaria para hacer atractiva la inversión en otro sector que éste abastece (encadenamiento hacia atrás) o procesa (encadenamiento hacia adelante). Así, toda actividad está encadenada con otras. Es la posible discontinuidad en el impacto de la decisión de inversión, que es crucial, pues la realización de una inversión hace rentable la generación de una segunda inversión y viceversa, y en tal situación, la toma de decisiones en forma coordinada asegura la rentabilidad de cada una de las inversiones.

En la teoría de la interacción y los distritos industriales, no existe un consenso absoluto sobre la definición de distrito industrial, por lo que se han manejado diferentes términos para denominarlos, tales como: agrupamientos empresariales, clusters, cooperación interempresarial. Para Conssentino et al. (1996), un distrito industrial es una aglomeración de cientos y a veces miles de empresas pequeńas y/o medianas orientadas a un determinado sector industrial y concentradas en la misma área. El concepto de "nuevos distritos industriales", señalan De Luca y Soto (1995), está basado en la reestructuración productiva: en respuesta a la emergencia de mercados globales y al cambio tecnológico rápido, las empresas se han visto obligadas a adoptar métodos de organización más flexibles, mayor flexibilidad también en su gestión y organización de los recursos humanos, en la adquisición de habilidades por parte de sus empleados, así como en la adopción de formas y métodos de interrelación con otras empresas. 
El desarrollo de capacidades de innovación. Un estudio del cluster de transporte en Aguascalientes, México / M. Feria

La teoría de la interacción pretende explicar las condiciones más propicias para que haya aprendizaje basado en la interacción, lo que, según Bianchi (1992), explicaría el éxito de los llamados "distritos industriales" en muchas regiones de Italia; dentro de la región de la Emilia Romagna el impulso fue de los empresarios a través de sus cámaras y asociaciones. Asimismo, la interacción acelera la difusión del conocimiento y la innovación, y genera el capital social que es un bien internalizado por el conjunto de empresas en el distrito. La interacción intensa en una localidad genera derrames tecnológicos y economías externas y de escala para el conjunto de empresas del distrito que no podrían ser internalizados de estar cada empresa interactuando con las otras a gran distancia.

Las economías externas (o eficiencia colectiva pasiva), son ahorros que una empresa puede realizar debido al accionar de otros actores, y surgen cuando estos actores no pueden incorporar todos los costos y beneficios de una actividad; esto puede ser el resultado de una investigación o una inversión en capital humano "se derrama" hacia otras empresas. Las economías externas no necesariamente requieren una concentración geográfica, pero la cercanía física de los actores complementarios suele facilitar la actividad. La especificidad de las actividades industriales de cada país, la identidad cultural y la estructura del mercado, dan origen a un conjunto de instituciones nacionales que funcionan como una externalidad dinámica en el proceso de innovación.

En este contexto, el trabajo que aquí se presenta pretende estimular tanto a los productores como a los administradores del desarrollo sobre las ventajas de construir los procesos de innovación, que dejan de ser un fenómeno de carácter individual y se transforman en un fenómeno colectivo en donde lo esencial se manifiesta tanto en la capacidad de interactuar y cooperar, como en la presencia de una estructura institucional adecuada y capaz de promover las actividades innovativas de los agentes económicos. La construcción de capacidades de innovación es un objetivo primordial puesto que las mismas comprenden relaciones de coordinación, cooperación y colaboración más intensas y sólidas, que ayuden a incorporar el conocimiento a los procesos, productos, gestiones e intercambios, para competir en un mundo globalizado. Así, la característica central, común a estas empresas (en el marco de un sistema innovativo), es su integración en estructuras reticulares (redes) para desarrollar capacidades tecnológicas, niveles de competitividad y relaciones de cooperación dentro de un espacio en que los agentes locales son los actores directos para crear un tejido productivo local común.

\section{Método}

El estudio es llevado a cabo a entre 2014 y 2015. El trabajo es un estudio de caso y se caracteriza por ser transversal, diagnóstico-descriptivo, realizado a través de un cues- 
tionario estructurado, con respuestas cerradas, mediante la escala de Likert (Ganzaroli et al., 2006). Los resultados alcanzados son producto de la metodología cuantitativa con una estrategia estadística no paramétrica para variables clasificatorias con nivel de medición nominal, en la que se usó la Rho de Spearman (Hernández et al., 2002), que permite ver el grado de relación respecto a las variables clave que tienen injerencia en la construcción de las capacidades de innovación y colaboración de las empresas en el entorno.

Para el acopio de la información se utilizó la técnica de encuesta, para lo cual se diseńó y aplicó un cuestionario al sector empresarial. Se optó por aplicar el total del universo de las empresas que integran el cluster del Transporte en Aguascalientes ( 25 empresas). Ello precisó el empleo de una prueba de correlación no paramétrica para variables clasificatorias. El instrumento incluyó 48 preguntas cerradas, distribuidas en seis apartados, en donde se incluyen aspectos relativos al tipo de relaciones que se tienen en el territorio, mercado, vinculación, cooperación, colaboración y competencia entre las empresas y con otras instituciones.

El objetivo del análisis estadístico se encaminó a describir, "medir" y evaluar los principales nexos que vinculan a las empresas que conforman el cluster de Transporte en Aguascalientes, con el objeto de determinar la capacidad de innovación ${ }^{1}$, las debilidades y fortalezas del Sistema de innovación en el estado, y el papel que ejercen en éstas los agentes del sistema.

Finalmente, es importante señalar que en los aspectos tácitos del proceso de aprendizaje existe entre otras cosas, una crisis de los métodos tradicionales de medición de las actividades innovativas, que en general miden variables de los aspectos formales del aprendizaje en las organizaciones; así se evalúan el gasto efectuado en los laboratorios de Investigación y Desarrollo, y el desarrollo de patentes, pero no se refleja la importancia de los procesos incrementales de innovación y que la capacidad innovativa está difundida a lo largo y a lo ancho de la organización (Boscherini y Yoguel, 1996; Moori-Koenig y Yoguel, 1998).

De esta manera, se parte de la idea de que la generación y circulación de conocimientos, tanto en el seno de la empresa como la existente entre instituciones, constituye un proceso complejo relacionado positivamente con la necesidad de resolver problemas en situaciones de incertidumbre, con la demanda de soluciones no fácilmente codifi-

1 Entendido como el cumplimiento eficiente (no sólo eficaz) de los actores del sistema de innovación para crear vínculos y tejer relaciones cooperativas interinstitucionales en orden a crear, adaptar, gestionar, intercambiar y difundir el conocimiento. 
El desarrollo de capacidades de innovación. Un estudio del cluster de transporte en Aguascalientes, México / M. Feria

cables, con el grado de desarrollo de las competencias de los recursos humanos de la empresa, la forma como se organiza el proceso de trabajo y con el grado de importancia que tiene para la empresa la interpretación, y adaptación del conocimiento codificado externo. Por lo tanto, el indicador de la capacidad innovativa de los agentes apunta a estimar, como mera aproximación, el grado de circulación del conocimiento a partir de vínculos formales e informales desarrollados con otros agentes e instituciones en el territorio en el que están localizados.

En este sentido, como lo mencionan Yoguel y Boscherini (2005), el indicador de la capacidad innovativa de las firmas debería incluir una evaluación de la influencia y de las externalidades producidas por el ambiente económico, social e institucional en el que las empresas desarrollan sus actividades. Así, la inclusión de variables que tomen en cuenta la cooperación que la institución desarrolla con otros agentes de su ámbito local puede constituir un indicador aproximado del "funcionamiento" del ambiente y de la calidad del sistema territorial.

Las variables fueron clasificadas considerando dos dimensiones específicas, referidas a precisar si se producen y qué tanto se producen las relaciones de cooperación, colaboración y competencia entre las empresas que integran el cluster y si realmente se generan las condiciones que produzcan procesos de innovación que generen un incremento en la capacidad innovativa. Una vez seleccionadas las variables se realizó el análisis factorial de componentes principales, a través del paquete estadístico SPSS con el fin de comprobar las variables que tienen mayor peso dentro del conglomerado.

Por tanto, el interés de fondo del análisis estadístico era especificar si se producen y qué tanto se producen las relaciones de cooperación, colaboración y competencia entre las empresas, los resultados obtenidos se contrastaron y enriquecieron con la literatura disponible, con información adicional proporcionada a través de entrevistas con algunos representantes de las empresas involucradas. A partir de estos elementos se construyó una interpretación global acerca de la manera en que se han generado o entorpecido las capacidades tecnológicas y el desempeño innovativo de los miembros del tejido institucional.

\section{Resultados}

El estado de Aguascalientes es el más claro ejemplo de desarrollo industrial planificado: hace aproximadamente tres décadas comenzó un proceso de transformación en el sistema productivo de la entidad en el cual la producción industrial sustituyó a la producción agrícola y artesanal. El proceso de transformación industrial en el estado afronta un nuevo reto: la globalización; este proceso presenta una situación fundamental y ante la cual nadie 
puede quedar exento de "la competencia", es por esto que las empresas locales deben buscar nuevas formas de producción, adaptarse a los nuevos paradigmas económicos y encontrar las estrategias adecuadas para hacer frente a la indiscriminada apertura comercial. La conformación de agrupamientos industriales o clusters ha sido una de las respuestas para poder hacer frente a la competencia buscando a través de la asociatividad mayores niveles de competitividad y por lo tanto de participación en los mercados.

En base al Censo económico 2014 realizado por el Instituto Nacional de Estadística, Geografía e Informática (INEGI, 2015), en el Estado de Aguascalientes predomina la microempresa en un $94,42 \%$ del total de empresas, 4,49\% la pequeña, 0,85\% la mediana y sólo el 0,22\% la gran empresa; de las cuales, el 9,85\% pertenece al sector industrial, el $44,79 \%$ al comercial y el $43,55 \%$ al de servicios y en otros sectores $1,78 \%$.

El Cluster del Transporte de Aguascalientes (CLUSTRAN) tiene relaciones con los siguientes sectores: Sector Gubernamental: Secretaría de Desarrollo Económico del Estado de Aguascalientes; Sector Educativo: Instituto Tecnológico y de Estudios Superiores de Monterrey, Universidad Bonaterra, A. C., Universidad Autónoma de Aguascalientes, Universidad Tecnológica y Universidad Interamericana para el Desarrollo.

En el año 2000 se constituye legalmente el Clustran (Cluster del Transporte) en un esfuerzo conjunto de la industria del transporte y el gobierno del estado así como de instituciones y organismos promotores de la cooperación interempresarial, la conformación de esta agrupación industrial se crea ante la necesidad de establecer nuevas formas de estructuración industrial que respondieran mejor a la competencia, a la búsqueda de calidad centrada en la cooperación y a la integración de estrategias conjuntas. Aspecto esencial en la elaboración del trabajo para la región de Aguascalientes, ya que éste es un entorno adecuado para explicar el papel que asumen los agentes locales en la creación de un tejido productivo local.

Por otra parte, como se mencionó supra, el cluster del transporte de Aguascalientes lo integró el gobierno del estado, como una estrategia de agrupamiento sectorial a fin de apoyar en un meso nivel y competir en el macro nivel.

En el ámbito del desarrollo de clusters y en este caso en específico el del transporte, lo que se detecta es que todavía se encuentra en una etapa de formación de relaciones y acoplamiento entre empresas y demás actores, en donde los actores gubernamentales tienen un papel fundamental para generar los mecanismos necesarios e indispensables mediante la construcción de políticas públicas. Como se demostró en otro trabajo (Feria, 2005), los enlaces establecidos siguen siendo débiles e incluso inexistentes en algunas Instituciones de Educación Superior en términos de redes de conocimiento que impliquen trabajo conjunto en investigación y desarrollo. 
El desarrollo de capacidades de innovación. Un estudio del cluster de transporte en Aguascalientes, México / M. Feria

Cuadro 1. Principales relaciones entre las interacciones de la empresa, el cliente y la apreciación del mercado. Correlaciones.

\begin{tabular}{|c|c|c|c|c|c|}
\hline & & & $\begin{array}{l}\text { Interacción de } \\
\text { la empresa con } \\
\text { el cliente }\end{array}$ & $\begin{array}{l}\text { Interacción del } \\
\text { cliente con la } \\
\text { producción }\end{array}$ & $\begin{array}{l}\text { Nivel de } \\
\text { apreciación del } \\
\text { mercado }\end{array}$ \\
\hline \multirow[t]{3}{*}{$\begin{array}{l}\text { Spearman's } \\
\text { rho }\end{array}$} & $\begin{array}{l}\text { Interacción de } \\
\text { la empresa con } \\
\text { el cliente }\end{array}$ & $\begin{array}{c}\text { Correlation } \\
\text { Coefficient } \\
\text { Sig. (2-tailed) } \\
\text { N }\end{array}$ & 1,000 & $\begin{array}{c}, 594^{*} \\
, 032 \\
25\end{array}$ & $\begin{array}{c}, 719^{* *} \\
, 006 \\
25\end{array}$ \\
\hline & $\begin{array}{l}\text { Interacción del } \\
\text { cliente con la } \\
\text { producción }\end{array}$ & $\begin{array}{l}\text { Correlation } \\
\text { Coefficient } \\
\text { Sig. (2-tailed) } \\
\mathrm{N}\end{array}$ & $\begin{array}{c}.594^{*} \\
\\
, 032 \\
25\end{array}$ & $\begin{array}{c}1,000 \\
. \\
25\end{array}$ & $\begin{array}{c}, 700^{* *} \\
, 008 \\
25\end{array}$ \\
\hline & $\begin{array}{l}\text { Nivel de } \\
\text { apreciación del } \\
\text { mercado }\end{array}$ & $\begin{array}{c}\text { Correlation } \\
\text { Coefficient } \\
\text { Sig. (2-tailed) } \\
\text { N }\end{array}$ & $\begin{array}{c}, 719^{* *} \\
, 006 \\
25\end{array}$ & $\begin{array}{c}, 700^{* *} \\
, 008 \\
25\end{array}$ & $\begin{array}{c}1,000 \\
25\end{array}$ \\
\hline
\end{tabular}

* Correlation is significant at the 0,05 level (2-tailed).

** Correlation is significant at the 0,01 level (2-tailed).

Como se puede observar en el cuadro 1, la variable "interacción de la empresa con el cliente" en las empresas encuestadas muestra una relación significativa $(r=0,594$ **, $p \leq 0,032$ ), entre la variable “interacción del cliente con la producción”. También la variable "interacción de la empresa con el cliente" con la variable "nivel de apreciación del mercado" se asocian de manera positiva pues el coeficiente de correlación es de $\left(r=0,719^{* *}, p \leq 0,006\right)$, la correlación es significativa con una $p$ menor que 0,01 a dos colas, situación importante porque denota que la interacción del cliente con la producción y la empresa con el cliente están en continua comunicación dentro del mercado.

Asimismo, lo anterior va de acuerdo con la variable "nivel de apreciación del mercado" con la variable "interacción del cliente con la producción" se asocian de manera positiva pues el coeficiente de correlación es de $\left(r=0,700^{* *}, p \leq 0,008\right)$, como se observa en el mismo cuadro.

En cuanto a las estrategias de producción de las empresas, la variable "planificación de la producción" con la variable "estrategias de producción" y ésta con la variable "puntualidad de la entrega" tienen una correlación positiva $0,785^{*}, 0,625^{*}$ y $0,591^{*}$ respectivamente $(p \leq 0,001 ; 0,022$ y 0,001$)$, lo que muestra una relación de concordancia entre lo que hacen y cómo funcionan; esto involucra tanto a la empresa, como a las demás empresas y los proveedores que las surten, pero, sin embargo, esta correlación es más bien baja, como se observa en el cuadro 2 . 
Cuadro 2. Relación entre estrategias de producción, planificación y puntualidad en la entrega. Correlaciones.

\begin{tabular}{|c|c|c|c|c|c|}
\hline & & & $\begin{array}{l}\text { Estrategia de } \\
\text { producción }\end{array}$ & $\begin{array}{l}\text { Planificación } \\
\text { de la } \\
\text { producción }\end{array}$ & $\begin{array}{l}\text { Puntualidad } \\
\text { en la entrega }\end{array}$ \\
\hline \multirow[t]{3}{*}{$\begin{array}{c}\text { Spearman's } \\
\text { rho }\end{array}$} & $\begin{array}{l}\text { Estrategia de } \\
\text { producción }\end{array}$ & $\begin{array}{c}\text { Correlation Coefficient } \\
\text { Sig. (2-tailed) } \\
\mathrm{N}\end{array}$ & $\begin{array}{c}1,000 \\
\dot{2} \\
25\end{array}$ & $\begin{array}{c}, 785^{* *} \\
, 001 \\
25\end{array}$ & $\begin{array}{c}, 625^{*} \\
, 022 \\
25\end{array}$ \\
\hline & $\begin{array}{l}\text { Planificación } \\
\text { de la } \\
\text { producción }\end{array}$ & $\begin{array}{c}\text { Correlation Coefficient } \\
\text { Sig. (2-tailed) } \\
\mathrm{N}\end{array}$ & $\begin{array}{c}, 785^{* *} \\
, 001 \\
25\end{array}$ & $\begin{array}{c}1,000 \\
\cdot \\
25\end{array}$ & $\begin{array}{c}, 591^{*} \\
, 033 \\
25\end{array}$ \\
\hline & $\begin{array}{c}\text { Puntualidad en } \\
\text { la entrega }\end{array}$ & $\begin{array}{c}\text { Correlation Coefficient } \\
\text { Sig. (2-tailed) } \\
\text { N }\end{array}$ & $\begin{array}{c}, 625^{*} \\
, 022 \\
25\end{array}$ & $\begin{array}{c}, 591^{*} \\
, 033 \\
25\end{array}$ & $\begin{array}{c}1,000 \\
\cdot \\
25\end{array}$ \\
\hline
\end{tabular}

* Correlation is significant at the 0,05 level (2-tailed).

** Correlation is significant at the 0,01 level (2-tailed).

Asimismo, la cultura empresarial pone obstáculos para establecer redes de colaboración y cooperación donde se participe de manera conjunta en el intercambio de información. Se observa que la mayoría de las empresas cuentan con limitado uso de planeación estratégica y conocimiento en el desarrollo organizacional, centrado en el dueño, con un estilo autoritario y con baja capacidad empresarial.

La falta de cultura de calidad, a su vez, no ha permitido el desarrollo de mercados de servicios de soporte; cabe seńalar que en cambio existe una cultura de no pago por servicios y la no facturación. Estas condiciones provocan que el desarrollo competitivo de la actividad de este sector esté limitado por falta de estructuras de mercado, falta de un mercado de demanda organizada de insumos y de canales de distribución eficientes, incluyendo un grupo de proveedores vinculados a los procesos de producción, y falta de liquidez, aunado a bajos recursos presupuestales destinados a la investigación y tecnología, cuya consecuencia se dirige a la falta de condiciones e incentivos para competir.

En las empresas estudiadas existen imperfecciones determinadas por asimetrías de información e indivisibilidades que afectan especialmente a las empresas de menor tamańo, pues su inserción en el tejido productivo es débil, y muestran además serios fraccionamientos por la falta de continuidad de los programas de apoyo.

Por otro lado, la relativa juventud de las instituciones que realizan investigación científica y tecnológica en Aguascalientes influye en la debilidad de su posición dentro del SRI, por la precariedad de los recursos económicos que se le destinan y la poca relevancia que la ciencia y la tecnología tienen en la cultura estatal. 
El desarrollo de capacidades de innovación. Un estudio del cluster de transporte en Aguascalientes, México / M. Feria

Cuadro 3. Rubros de actividades de innovación y su relación con la capacitación de trabajadores. Correlaciones.

\begin{tabular}{|c|c|c|c|c|c|}
\hline \multicolumn{2}{|c|}{} & $\begin{array}{c}\text { Rubros en que } \\
\text { se desarrollaron } \\
\text { actividades de } \\
\text { innovación }\end{array}$ & $\begin{array}{c}\text { Resultados } \\
\text { obtenidos por } \\
\text { el desarrollo de } \\
\text { actividades de } \\
\text { innovación }\end{array}$ & $\begin{array}{c}\text { Rubro de } \\
\text { capacitación } \\
\text { recibido por } \\
\text { trabajadores }\end{array}$ \\
\hline $\begin{array}{c}\text { Spearman's } \\
\text { rho }\end{array}$ & $\begin{array}{c}\text { Rubros en que } \\
\text { se desarrollaron } \\
\text { actividades de } \\
\text { innovación }\end{array}$ & $\begin{array}{c}\text { Correlation } \\
\text { Coefficient } \\
\text { Sig. (2-tailed) } \\
\mathrm{N}\end{array}$ & 1,000 &, $902^{* *}$ &, 545 \\
\cline { 2 - 7 } & $\begin{array}{c}\text { Resultados obtenidos } \\
\text { por el desarrollo } \\
\text { de actividades de } \\
\text { innovación }\end{array}$ & $\begin{array}{c}\text { Correlation } \\
\text { Coefficient } \\
\text { Sig. (2-tailed) } \\
\mathrm{N}\end{array}$ &, 25 &, 001 &, 054 \\
& $\begin{array}{c}\text { Rubro de } \\
\text { capacitación recibido } \\
\text { por trabajadores }\end{array}$ & $\begin{array}{c}\text { Correlation } \\
\text { Coefficient } \\
\text { Sig. (2-tailed) } \\
\mathrm{N}\end{array}$ & 25 & 1,000 & 25 \\
\hline
\end{tabular}

** Correlation is significant at the 0.01 level (2-tailed).

El cuadro 3 presenta el análisis de las empresas de la región acerca de la variable "resultados obtenidos por el desarrollo de actividades de innovación" con la variable "rubro de capacitación recibida por trabajadores" muestra una relación significativa $\left(r=0,688^{* *}\right.$, $p \leq 0,009)$. Asimismo si en este tipo de empresas se destaca el desarrollo de actividades de innovación con el rubro de capacitación recibido por los trabajadores sería sólo una parte de lo que abarca el rubro de actividades de innovación, por lo que este tipo de actividades no han sido lo suficientemente impactantes para generar las externalidades positivas que necesita la región.

Por otra parte, se realizó un análisis factorial como herramienta para identificar los componentes adecuados para las empresas que conforman el cluster. De acuerdo con Hair et al. (1999:776) "el análisis factorial es un método estadístico multivariante que permite definir la estructura subyacente en una matriz de datos", es decir, permite analizar las correlaciones entre un gran número de variables que se correlacionan altamente entre sí. Así, el uso de este método consiste en identificar las variables que tienen más valor dentro del cluster (ver cuadro 4). 
Cuadro 4. Variables utilizadas en el análisis del Cluster.

\begin{tabular}{|l|l|}
\hline 1 Interacción de la empresa con el cliente & 10 Sistemas informativos \\
\hline 2 Interacción del cliente con la producción & 11 Capacidad de desarrollar nuevos productos \\
\hline 3 Relevancia tecnología sist. prod. loc. & 12 Capacidad de desarrollar rápidamente productos \\
\hline 4 Relevancia tecnología sist. prod. ext. & $\begin{array}{l}13 \text { Capacidad de utilizar información con } \\
\text { proveedores }\end{array}$ \\
\hline 5 Relevancia investigación sist. prod. loc. & 14 Capacidad de identificar proveedores idóneos \\
\hline 6 Relevancia invest. sist, prod. ext. & 15 Capacidad de generar alianzas con proveedores \\
\hline 7 Relev. serv. informativos sist. prod. ext. & $\begin{array}{l}16 \text { Inversión en tecn. de inf. para relación con } \\
\text { proveedores }\end{array}$ \\
\hline $\begin{array}{l}\text { 8 Modalidad en que la empresa adquiere } \\
\text { aprendizaje }\end{array}$ & $\begin{array}{l}17 \text { Inversión en tecnología de información para } \\
\text { mantener clientes }\end{array}$ \\
\hline 9 Innovación, investigación y desarrollo & \\
\hline
\end{tabular}

Fuente: elaboración propia.

Para tal efecto se utilizó el método de componentes principales y rotación varimax. En la revisión del sistema se dio una reducción de los componentes en cinco factores que explicaban el 86,79 por ciento de la varianza (ver cuadro 5), las saturaciones máximas para cada ítem se presentan en el mismo cuadro 5.

Cuadro 5. Varianza total explicada (autovalores iniciales).

\begin{tabular}{|c|l|c|c|c|c|c|}
\hline \multirow{4}{|c|}{ Autovalores iniciales } & \multicolumn{3}{c|}{$\begin{array}{c}\text { Suma de saturaciones al } \\
\text { cuadrado de la extracción }\end{array}$} \\
\hline Comp. & \multicolumn{1}{|c|}{ Total } & \% de la varianza & \% acumulado & Total & $\begin{array}{c}\text { \% de la } \\
\text { varianza }\end{array}$ & $\begin{array}{c}\% \\
\text { acumulado }\end{array}$ \\
\hline 1 & 6,17203997238275 & 34,289110957682 & 34,289110957682 & 6,172 & 34,289 & 34,289 \\
\hline 2 & 3,9302393773421 & 21,8346632074561 & 56,1237741651381 & 3,930 & 21,835 & 56,124 \\
\hline 3 & 3,02306986947845 & 16,7948326082136 & 72,9186067733517 & 3,023 & 16,795 & 72,919 \\
\hline 4 & 1,43844247494407 & 7,99134708302259 & 80,9099538563743 & 1,438 & 7,991 & 80,910 \\
\hline 5 & 1,05879931361919 & 5,88221840899551 & 86,7921722653698 & 1,059 & 5,882 & 86,792 \\
\hline 6 & 0,691768707120621 & 3,84315948400345 & 90,6353317493733 & & & \\
\hline 7 & 0,579656194225426 & 3,22031219014126 & 93,8556439395145 & & & \\
\hline 8 & 0,435910925242187 & 2,42172736245659 & 96,2773713019711 & & & \\
\hline 9 & 0,352318209517851 & 1,95732338621028 & 98,2346946881814 & & & \\
\hline 10 & 0,15882395673642 & 0,88235531520233 & 99,1170500033837 & & & \\
\hline 11 & 0,122945799001276 & 0,683032216673757 & 99,8000822200575 & & & \\
\hline 12 & 0,0359852003896553 & 0,199917779942529 & 100 & & & \\
\hline
\end{tabular}

Fuente: elaboración propia. 
El desarrollo de capacidades de innovación. Un estudio del cluster de transporte en Aguascalientes, México / M. Feria

Como se aprecia en el cuadro 6, las comunalidades son próximas a la unidad, por lo que se garantiza un alto grado de fiabilidad en los resultados. La variable que muestra la comunalidad más alta es "Relevancia Investigación sistema productivo externo" con ,984, seguida de la comunalidad "Modalidad en que la empresa adquiere aprendizaje" con ,966 y la más baja "Innovación, Investigación y Desarrollo" con ,5808.

Cuadro 6. Comunalidades.

\begin{tabular}{|l|c|l|}
\hline \multicolumn{1}{|c|}{ Variables } & Inicial & \multicolumn{1}{c|}{ Extracción } \\
\hline Interacción de la empresa con el cliente & 1,000 & 0,920624091726146 \\
\hline Relevancia Investigación sist. prod. ext. & 1,000 & $0,984184444652485(1)$ \\
\hline Interacción del cliente con la producción & 1,000 & 0,7862904655572878 \\
\hline Relevancia tecnología sist. prod. loc. & 1,000 & 0,91229827790486 \\
\hline Relevancia tecnología sist. prod. ext. & 1,000 & 0,912709202839096 \\
\hline Relevancia investigación sist. prod. loc. & 1,000 & 0,904462146999014 \\
\hline Relevancia serv. informativos sist. prod. ext. & 1,000 & 0,832831036246774 \\
\hline Modalidad en que la empresa adquiere aprendizaje & 1,000 & $0,96610235646744(2)$ \\
\hline Importancia de la protección del conocimiento & 1,000 & 0,845182149560468 \\
\hline Innovación, investigación y desarrollo & 1,000 & $0,580849051621851(3)$ \\
\hline Sistemas informativos & 1,000 & 0,895298118811217 \\
\hline Capacidad de desarrollar nuevos productos & 1,000 & 0,826873137187599 \\
\hline Capacidad de desarrollar rápidamente productos & 1,000 & 0,936879624680955 \\
\hline Capacidad de utilizar información con proveedores & 1,000 & $0,738246937857174(4)$ \\
\hline Capacidad de identificar proveedores idóneos & 1,000 & 0,869888260430493 \\
\hline Capacidad de generar alianzas con proveedores & 1,000 & 0,915126816159002 \\
\hline Inversión en tecn. de inf. para relación con proveedores & 1,000 & 0,898563976037817 \\
\hline Inversión en tecn. de inf. para mantener clientes & 1,000 & 0,896180913011296 \\
\hline
\end{tabular}

Fuente: elaboración propia.

La débil integración y articulación con el conjunto de actividades industriales crea un círculo vicioso, cuyas características, referidas a la capacidad de innovación, remiten a la mera adaptación de tecnologías desarrolladas a nivel internacional.

Así, el potencial para apropiarse de conocimientos tecnológicos e innovativos por parte de subsectores que dependen de capitales externos es muy limitado, pues está supeditado casi exclusivamente a las fuentes de cada sector, en donde la cooperación intersectorial es reducida, lo que también limita las redes de conocimiento interno y debilita el funcionamiento del Sistema productivo local. De hecho los beneficiarios de los incentivos de políticas públicas en su mayoría son empresas grandes y transnacionales. 
A pesar de todo, una de las grandes ventajas que ha tenido Aguascalientes es el haber permitido, por los ámbitos público y privado, la aplicación de programas flexibles de acuerdo con las competencias tecnológicas y las necesidades industriales en los distintos niveles y en donde la consolidación de los clusters forma parte de las estrategias a nivel de planeación regional.

\section{Conclusiones}

1. Si bien una red articulada entre las empresas que integran el cluster propicia un ambiente interinstitucional favorable para el desarrollo de la innovación y el aprendizaje, persisten múltiples problemas de coordinación. La falta de información, la duplicación de actividades y, sobre todo, la falta de motivación entre los empresarios para apoyar, solicitar y colaborar en programas de capacitación, impiden consolidar un comportamiento asociativo entre la industria y el sistema educativo técnico que permita utilizar productivamente estos servicios.

2. En el trabajo se identificaron factores como recursos que contribuyen a la innovación en las empresas. La identificación de estos factores permite definir renglones que hay que apoyar en la región para impulsar un mejor desarrollo de la capacidad innovadora.

3. El objetivo del trabajo fue tratar de identificar la relación que existe entre el nivel de integración del cluster del transporte de Aguascalientes y el nivel de desarrollo del mismo en cuanto a su forma de operar y vincularse dirigiendo sus esfuerzos hacia la innovación y la competitividad; en este se detectó que sí existe relación entre las variables pero que la manera de funcionar dista mucho de la manera de vincularse con otras empresas lo que obstaculiza el grado de integración y desarrollo de los mismos.

4. La respuesta a la hipótesis inicial fue validada al establecerse una correlación significativa positiva entre las variables, sin embargo, se encuentra también un comportamiento desigual de las empresas estudiadas.

5. A pesar de cierto avance en el desempeńo innovativo del tejido interinstitucional, el interés por parte de los empresarios hacia el establecimiento de relaciones con el sector educativo y con instituciones de ciencia y tecnología no es suficiente. Con todo, se acepta en el discurso que el fortalecimiento y la solidez de estos vínculos son relevantes y necesarios para generar un proceso de innovación mayor en la región y un incremento en la capacidad innovativa.

6. Las variables más significativas dentro del modelo de componentes principales y la rotación varimax son cinco, que muestran las saturaciones máximas, siendo por 
El desarrollo de capacidades de innovación. Un estudio del cluster de transporte en Aguascalientes, México / M. Feria

orden de importancia: "Relevancia de Investigación sistema productivo externo", "Modalidad en que la empresa adquiere aprendizaje", "Capacidad de desarrollar rápidamente productos", "Interacción de la empresa con el cliente" y "Capacidad de generar alianzas con proveedores".

7. Los factores diferenciadores de la capacidad innovativa de los agentes son los esfuerzos de capacitación, la proporción de ingenieros en los equipos de desarrollo y el grado de alcance de sus actividades, estos factores junto con la escasa cooperación tecnológica local, ponen de manifiesto que los procesos de innovación se sustentan fundamentalmente en esfuerzos individuales de las empresas, como lo mencionan en su estudio Yoguel y Boscherini (2001), en el que catalogan al ambiente como negativo por la proporción tan baja de ingenieros y técnicos en el equipo de desarrollo de nuevos productos y por el grado de cooperación tecnológica formal e informal dentro de las empresas.

8. La relativa juventud de las instituciones que realizan investigación científica y tecnológica en Aguascalientes influye en la debilidad de su posición dentro del estado, por la precariedad de los recursos económicos que se le destinan y la poca relevancia que la ciencia y la tecnología tienen en la cultura estatal, tal es así que dentro de la comunalidad más baja dentro del modelo se encuentra la variable "Innovación, Investigación y Desarrollo".

9. Esto responde a otro cuestionamiento básico de la investigación, la relación que existe entre el nivel de integración de las empresas que conforman el cluster del transporte de Aguascalientes y el nivel de desarrollo de las mismas en cuanto a su forma de operar y vincularse dirigidos hacia la innovación y competitividad, se puede decir que aunque existe una relación positiva, esta relación es precaria; se detectan asimetrías de información y de adaptación o renovación de tecnologías en el mejor de los casos y no se construye el desarrollo de investigación básica de interés para las empresas, o en su caso este desarrollo es muy escaso.

10. El objetivo de la presente investigación fue contribuir al estudio de las capacidades de innovación en una región como lo es el estado de Aguascalientes y para ganar validez debe replicarse el estudio en los diferentes estados de la República Mexicana.

11. Así, para aprovechar las externalidades generadas por un ambiente "local" se necesitan ciertas competencias mínimas sin las cuales es imposible complementar los conocimientos faltantes, disminuir las incertidumbres dinámicas, potenciar los procesos de aprendizaje y contrarrestar las debilidades de la cultura organizativa. 


\section{Bibliografía}

Bianchi, Patrizio (1992). "Competencia dinámica, distritos industriales y medidas locales”. Serie Industrialización y Desarrollo Tecnológico, No.13, LC/G.1752, Santiago de Chile, CEPAL.

Boscherini, Fabio y Yoguel, Gabriel (1996). Innovative processes in SMEs: some considerations about the Argentine case. CEPAL-IDCJ, Buenos Aires.

Boscherini, Fabio y Poma, Lucio (Comp.) (2000). "Más allá de los distritos industriales: el nuevo concepto de territorio en el marco de la economía global”. En Territorio, conocimiento y competitividad de las empresas. El rol de las instituciones en el espacio global (pp. 23-38).Buenos Aires: Miño y Dávila (Eds.)

Cossentino, Francesco; Pyke, Frank y Sengenberger, Werner (1996). Local and regional response to global pressure. The case of ltaly: industrial districts. Suiza: OIT.

De Luca, José Alberto y Soto, Gloria (1995). Los distritos industriales como estrategia de desarrollo regional. España: Ed. Caja Murcia.

Dosi, Giovanni et al. (2000). "Introduction: The nature and dynamics of organizational capabilities". En Dosi, G.; Nelson, R. y Winter, S. (Eds). The nature and dynamics of organizational capabilities. Gran Bretaña: Oxford University Press.

Ernest, D. y Lundvall, A. (1997). Information tecnology in the learning economy, challenges for developing countries. Danish Research Unit for Industrial Dynamics (DRUID), Working Paper No. 97-12.

Feria Cruz, Maribel (2005). Redes interinstitucionales, la construcción de un sistema de innovación en Aguascalientes, México. UAA-ITA, 1ª Edición.

Ganzaroli, Andrea; Fiscato, Gianluca y Pilotti, Luciano (2006). "A scoreboard to evaluate clusters' competitiveness in the knowledge-based economy. an empirical study on emiliaromagna region". Working paper 30 (10), Universit' adegli studi di Milano, dipartimento di scienze economiche, aziendali e statistiche.

Hair, Joseph F.; Anderson, Rolph; Tatham, Ronald L. y Black, William (1999). Análisis multivariante. Madrid: Prentice Hall.

Hernández, Roberto; Fernández, Carlos y Baptista, Pilar (2002). Metodología de la investigación. México: Ed. McGraw-Hill.

Herrera, José (2006). Análisis de distritos o clusters industriales. http://www.econlink. com.ar/economia/clusters/clusters.shtml recuperado 11 de diciembre 2006.

Hirschman, O. Albert (1958). The Strategy of Economic Development, New Haven: Yale University Press. Edición en español: La estrategia del desarrollo económico, México, FCE, 1961. 
El desarrollo de capacidades de innovación. Un estudio del cluster de transporte en Aguascalientes, México / M. Feria

INEGI (2015). Censos económicos. Consultado en: http://www.inegi.org.mx/est/contenidos/proyectos/ce/ce2014/, 20 de noviembre del 2006 consultado el 5 de marzo de 2016.

Leonard, Barton L. D. (1995). Wellsprings of knowledge: building and sustaining the sources of innovation. Harvard Business School Press, Boston, Massachusetts. Paperback edition en 1998.

Marshall, Alfred (1920). Principles of Economics (8th Ed. 1920: 1st Ed. 1890). English Language Book Society, London.

Meyer-Stamer, Jörg y Harmes-Liedtke, Ulrich (2005). Como promover clusters. En: http://www.mesopartner.com/publications/mp-wp8_cluster_s. pdf, 2 de octubre del 2006.

Moori-Koening V. y Yoguel, G. (1998). "Capacidades innovadoras en un medio de escaso desarrollo del sistema local de innovación”. Comercio Exterior, Política industrial, innovación y empresas pequeñas I, México. 48. (8) 641-658.

OECD (1999). Managing National Innovation Systems. Paris: OECD.

OECD (1992). The technology and the economy. The key relationships, París: Organization for Economic Co-operation and Development.

Osorio Ramírez, Efrén (2006). Metodología para detección e identificación de clusters industriales. Edición electrónica. Texto completo en www.eumed.net/ libros/2006b/eaor/

Perego, Luis Héctor (2003). Competitividad a partir de los agrupamientos industriales. Un modelo integrado y replicable de clusters productivos. Argentina. http://www. eumed.net/cursecon/libreria/lhp/lp-agrupa.doc.10 de octubre del 2006.

Porter, Michael (1991). La ventaja competitiva de la naciones. Argentina, Vergara ediciones.

Porter, Michael (1998). Ventaja competitiva creación y sostenimiento de un desempeño superior. México. Ed. Continental.

Rabellotti, Roberta (1998). "Recovery of a Mexican Cluster: Devaluation Bonanza or Collective Efficiency?”. Working paper 71, Institute of Development Studies, England.

Ramos, Joseph (1998). "Una estrategia de desarrollo a partir de los complejos productivos (clusters) en torno a los recursos naturales". Revista de la CEPAL, núm. 66, pp. 4-125, diciembre 1998.

Storper, Michael (1997). The regional world. Territorial development in a global economy, New York, U.S.A., The Guilford Press. 
Scott, Alleny y Storper, Michael (2003). "Regions, globalization, development". Regional Studies, 37, 6\&7, pp. 579-93.

Teece, David J. et al. (2000). "Dynamic Capabilities an Strategic Management" en Giovanni Dosi, Richard R. Nelson y Sidney G. Winter (editores) The Nature and dynamics of organizational capabilites, Oxford University Press, Gran Bretaña.

Yoguel, Gabriel y Boscherini, Fabio (2005). "El desarrollo de las capacidades innovativas de las firmas y el rol del sistema territorial”. En Casalet, Mónica; Cimoli, Mario y Yoguel, Gabriel (Compiladores). Redes, jerarquias y dinámicas productivas. Buenos Aires, Argentina. Miño y Dávila, srl-Flacso, México-OIT.

\section{Cómo citar este artículo:}

Feria, M. (2016). "El desarrollo de capacidades de innovación. Un estudio del cluster de transporte en Aguascalientes, México", Oikos No 41, 145-165, Escuela de Administración y Economía, Universidad Católica Silva Henríquez (UCSH), Santiago de Chile.

[http://ediciones.ucsh.cl/revistas.php]

Fecha de recepción: 11/03/2016

Fecha de aceptación: 17/05/2016 